\title{
Prevalence of anxiety among patients with irritable bowel syndrome: A pilot study
}

\author{
Lokugamage DR ${ }^{1}$, KodikaraArachchi RWK ${ }^{1}$, Lekamwasam JDVC ${ }^{1}$, Kumarasena S $^{1}$, De Silva PV ${ }^{2}$ \\ ${ }^{\text {I} T e a c h i n g ~ H o s p i t a l ~ K a r a p i t i y a, ~ G a l l e, ~ S r i ~ L a n k a . ~}$ \\ ${ }^{2}$ Department of Community Medicine, Faculty of Medicine, University of Ruhuna, Galle, Sri Lanka.
}

Correspondence: Dr. Don Ravindra Lokugamage

Teaching Hospital Karapitiya, Galle, Sri Lanka.

e-mail: rvndrlokugamage1@gmail.com

\begin{abstract}
Introduction: Aetiological factors of irritable bowel syndrome [IBS] among Sri Lankans are not well known. This study was carried out to find the prevalence of anxiety among patients with IBS and its association with symptoms of the disease.

Methods: A group of patients numbering 104 with IBS, presented to Medical and Gastroenterology Units and diagnosed according to the ROME III criteria were assessed with a combination of Generalised Anxiety Disorder-7 questionnaire (GAD) and Public Health Questionnaire-4 (PHQ). This data were compared with 104 age and sex matched controls.

Results: Majority (55.8\%) of IBS patients were females. Mean age of them was 46.9 years with a SD of $15.1 \mathrm{yrs}$ (range: 20-76). Majority (53.8\%) of IBS patients belonged to Social Class 5. Features of anxiety were found in $26 \%$ of IBS patients according to the PHQ and $43.3 \%$ according to the GAD. Only 3 controls $(2.88 \%)$ were detected to have anxiety according to the PHQ. Anxiety among controls according to the GAD was $4.8 \%$.

Conclusions: There is a higher occurrence of anxiety among patients with IBS when compared with age and sex matched controls. Whether this is a primary etiological factor or a secondary phenomenon due to the chronicity of the disease is unknown to us.
\end{abstract}

Keywords: Anxiety, Irritable Bowel Syndrome, Sri Lanka

\section{Introduction}

Irritable bowel syndrome (IBS) is a gastrointestinal syndrome characterized by chronic abdominal pain and altered bowel habits in the absence of any organic cause. In Sri Lanka no data are available on the prevalence of irritable bowel syndrome. However, it is not an uncommon condition in clinical practice. The prevalence of IBS in North America estimated from population-based studies is approximately 10 to $15 \%$ (1-6). A similar prevalence $(11.5 \%)$ has been reported from Europe as well.
In subsequent studies, however, the prevalence varied widely among countries (7).

IBS affects men and women, young and the elderly. However, younger patients and women are more likely to suffer from IBS (3). A systematic review estimated that there is an overall 2:1 female predominance in North America (3).

IBS accounts for a significant number of visits to primary care physicians and is the second highest cause of work absenteeism after the common cold (8). IBS is associated with increased health care costs 
with some studies suggesting annual direct and indirect costs of up to $\$ 30$ billion (9).

Aetiology of IBS is not clearly understood. Several factors are thought to be responsible for the disease. Gastrointestinal motility, visceral hypersensitivity, intestinal inflammation, infections, alterations in faecal microflora, food allergy, genetics factors, and psychosocial dysfunctions are some of them. Psychosocial factors may influence the expression of IBS (10). In a study of patients with IBS or non-ulcer dyspepsia, patients with GI symptoms reported more lifetime and daily stressful events than control groups (11). Another study found that compared with controls, patients with IBS exhibit increased anxiety, depression, phobias, and somatization (12). In a prospective study, psychosocial factors (anxiety, sleep problems, somatic symptoms) were shown to be independent risk factors for the development of IBS in a population not previously diagnosed with the condition (13).

Finding the aetiology of a disease will help to treat patients effectively. Because of this reason we decided to study the relationship between anxiety disorders and IBS. This study was carried out to find the prevalence of anxiety among patients with IBS and its association with symptoms of the disease.

\section{Methods}

Written permission to carry out the study was obtained from the Director of Teaching Hospital, Karapitiya. Ethical clearance for the study was obtained from the Ethics Committee of the Faculty of Medicine, University of Ruhuna, Galle, prior to data collection. Data collection was carried out after obtaining informed consent from the study subjects. The data obtained was treated confidentially. Comparative descriptive cross-sectional study design was used.

A group of 104 patients with IBS presented to Medical and Gastroenterology Units and diagnosed according to ROME III criteria were interviewed. We included only adult patients aged between 18-50 years and patients with the history of disease for more than 1year period. Patients on treatment for psychiatric disorder or on drugs that can potentially change the symptoms of anxiety and patients practicing non-pharmacological measures targeting anxiety reduction (Yoga, Meditation etc) were excluded. Hospital-based 104 age and sex-matched healthy controls were recruited for the study. They were selected among patients visiting the same hospital for reasons other than health issues.

Interviewer administered questionnaire was used as the study instrument. It included socio-demographic data, data on occupation related factors and Generalized Anxiety disorder 7 questionnaire and Public Health Questionnaire-4. Principal investigator collected data to ensure the completeness and accuracy of data.

All data were coded and entered into a database and cleaning and checking were done. Where appropriate, data were expressed as means and standard deviations. Differences between proportions of groups were tested for statistical significance using the Chi-square test. Probability values less than 0.05 were considered as statistically significant.

\section{Results}

We interviewed 104 subjects with the disease and 104 age and sex matched controls. Majority (55.8\%) of IBS patients were females. Mean age of them was 46.9 years with a Standard Deviation of 15.1 (range: 20 to 76 years) (Table 1). Majority $(53.8 \%)$ of patients belonged to the Social Class 5 (Table 1). Distribution of duration of disease among patients is shown in Table 2.

PHQ identified $26 \%$ of patients with IBS to have anxiety while according to the GAD $43.3 \%$ had features of anxiety (Table 3). Only 3 controls $(2.88 \%)$ were detected to have anxiety according to the PHQ. Anxiety among healthy controls according to GAD was $4.8 \%$ (Table 3). There was a higher prevalence of anxiety detected according to PHQ among those with IBS when compared with healthy controls (Table 4). Similarly, there was a higher prevalence of anxiety detected according to GAD among those with IBS when compared with study controls (Table 5). There was no statistically significant association of gender, age, social class or duration of disease with anxiety according to the PHQ or GAD ( $p>0.05)$. 
Table 1: Age and social class distribution among study participants with the disease $(n=104)$.

\begin{tabular}{lcc}
\hline Socio demographic variable & Number & Percentage \\
\hline Age & & \\
40 years or less & 38 & $36.5 \%$ \\
41 to 60 years & 41 & $39.5 \%$ \\
61 years and above & 25 & $24.0 \%$ \\
\hline Social class & & \\
Social class 1 & 4 & 3.8 \\
Social class 1 & 3 & $2.9 \%$ \\
Social class 1 & 26 & $25.0 \%$ \\
Social class 1 & 15 & $14.4 \%$ \\
Social class 1 & 56 & $53.9 \%$ \\
\hline
\end{tabular}

Table 2: Distribution of the duration of disease among patients with IBS

\begin{tabular}{lll}
\hline Duration of disease in months & Number & Percentage \\
\hline 12 months or less & 28 & $26.9 \%$ \\
13 to 36 months & 37 & $35.6 \%$ \\
More than 36 months & 39 & $37.5 \%$ \\
\hline Total & $\mathbf{1 0 4}$ & $\mathbf{1 0 0 \%}$ \\
\hline
\end{tabular}

Table 3: Anxiety among cases and controls according to GAD.

\begin{tabular}{lllll}
\hline \multirow{2}{*}{$\begin{array}{l}\text { Anxiety according to } \\
\text { GAD }\end{array}$} & \multicolumn{2}{c}{ Among cases } & \multicolumn{2}{c}{ Among controls } \\
\cline { 2 - 5 } & Frequency & Percent & Frequency & Percent \\
\hline No Anxiety & 59 & $56.7 \%$ & 99 & $95.2 \%$ \\
Mild Anxiety & 37 & $35.6 \%$ & 5 & $4.8 \%$ \\
Moderate Anxiety & 6 & $5.8 \%$ & 0 & $0 \%$ \\
Severe Anxiety & 2 & $1.9 \%$ & 0 & $0 \%$ \\
\hline Total & $\mathbf{1 0 4}$ & $\mathbf{1 0 0 . 0} \%$ & $\mathbf{1 0 4}$ & $\mathbf{1 0 0 . 0 \%}$ \\
\hline
\end{tabular}

Table 4: Anxiety detected by PHQ among those with and without IBS

\begin{tabular}{lcc}
\hline Disease Status & \multicolumn{2}{c}{ Anxiety according to PHQ } \\
\cline { 2 - 3 } & Anxiety positive & Anxiety negative \\
\hline With the disease & 27 & 77 \\
Without the disease & 3 & 101 \\
\hline Chi square value (Yates corrected) & $20.61 \mathrm{p}<0.001$ &
\end{tabular}


Table 5: Anxiety detected by GAD among those with and without IBS

\begin{tabular}{lcc}
\hline Disease Status & \multicolumn{2}{c}{ Anxiety according to GAD } \\
\cline { 2 - 3 } & Anxiety positive & Anxiety negative \\
\hline With the disease & 45 & 59 \\
Without the disease & 5 & 99 \\
\hline Chi square value (Yates corrected) & 40.05 & $\mathrm{p}<0.001$
\end{tabular}

\section{Discussion}

IBS is commonly regarded as a functional disorder and is hypothesised to be associated with anxiety. In current clinical practice we see a reasonable number of patients with IBS. Most of them are in younger age. But majority of these patients are not satisfied with the available treatment options. If we can find the aetiology it will be easy to control this condition.

The aetiology of IBS is not clearly understood. Several factors are thought to be responsible for the disease. Many aspects including genetic and environmental factors have been studied to find out the exact aetiology of the diseases but none have been proven.

Only few studies have addressed both anxiety and depression together. To measure the anxiety level we used GAD-7 and PHQ-4 questioners. All patients recruited were diagnosed according to Rome III criteria. All the patients who fulfilled the criteria presented to medical and Gastroenterology clinics were recruited during the data collection period of six months. Almost all the patients had negative colonoscopy.

According to a systematic review and meta-analysis by Guillaume Fond et al, patients with IBS have significantly higher levels of anxiety (14). This finding is in keeping with our results. Study published by Mariette Bengtsson et al, also reported a higher prevalence of anxiety among patient with IBS (15). According to Kabra N, et al, the prevalence of anxiety among patients with IBS was $31.4 \%$ (16). However this was a clinic based study and they have used Hamilton Anxiety rating scale instead of PHQ-4 or GAD. In an Iranian study by Modabbemia MJ et al, including 256 patients with IBS, $74.2 \%$ prevalence of Anxiety / Depressive problems was reported (17). But this study included depression too and this probably contributed to the relatively higher value. Study conducted by Lee $\mathrm{S}$ et al, from the Department of psychiatry in the Hong Kong University found that generalized anxiety disorder is five times more common in IBS patients than non-IBS respondents (18).

In conclusion we have found that there is a significant association between IBS and anxiety. Whether this is an aetiological factor or a consequence of the chronic nature of the disease is not clear to us. We suggest assessing anxiety level in IBS patients and taking appropriate actions to control anxiety level in these patients.

\section{Acknowledgements}

Professor Sarath Lekamwasam, Dean, Faculty of Medicine, Galle, Sri Lanka. Dr. M.M. Jeewantha. Dr. T.P.E. Oshadhi.

\section{References}

1. Talley NJ, Zinsmeister AR, Van Dyke C, Melton LJ $3^{\text {rd }}$. Epidemiology of colonic symptoms and the irritable bowel syndrome. Gastroenterology 1991; 101: 927.

2. Drossman DA, Li Z, Andruzzi E, et al. U.S. householder survey of functional gastrointestinal disorders. Prevalence, socio-demography, and health impact. Dig Dis Sci 1993; 38: 1569.

3. American College of Gastroenterology Task Force on Irritable Bowel Syndrome, Brandt LJ, Chey WD, et al. An evidence-based position statement on the management of irritable bowel syndrome. Am J Gastroenterol 2009; 104 Suppl 1: S1.

4. Hahn BA, Saunders WB, Maier WC. Differences between individuals with self-reported irritable bowel syndrome (IBS) and IBS-like symptoms. Dig Dis Sci 1997; 42: 2585. 
5. Saito YA, Locke GR, Talley NJ, et al. A comparison of the Rome and Manning criteria for case identification in epidemiological investigations of irritable bowel syndrome. Am J Gastroenterol 2000; 95: 2816.

6. Thompson WG, Irvine EJ, Pare P, et al. Functional gastrointestinal disorders in Canada: first population-based survey using Rome II criteria with suggestions for improving the questionnaire. Dig Dis Sci 2002; 47: 225.

7. Hungin AP, Whorwell PJ, Tack J, Mearin F. The prevalence, patterns and impact of irritable bowel syndrome: an international survey of 40,000 subjects. Aliment Pharmacol Ther 2003; 17: 643 .

8. Schster MM. Diagnostic evaluation of the irritable bowel syndrome. Gastroenterol Clin North Am 1991; 20: 269.

9. Sandler RS, Everhart JE, Donowitz M, et al. The burden of selected digestive diseases in the United States. Gastroenterology 2002: 122: 1500.

10. Chang L. The role of stress on physiologic responses and clinical symptoms in irritable bowel syndrome. Gastroenterology 2011; 140: 761.

11. Locke GR $3^{\text {rd }}$, Weaver AL, Melton LJ $3^{\text {rd }}$, Talley NJ. Psychosocial factors are linked to functional gastrointestinal disorders: a population based nested case-control study. $\mathrm{Am}$ J Gastroenterol 2004; 99:350.

12. Solmaz M, Kavuk I, Sayar K. Psychological factors in the irritable bowel syndrome. Eur JMed Res 2003; 8: 549.
13. Nicholl BI, Halder SL, Macfarlane GJ, et al. Psychosocial risk markers for new onset irritable bowel syndrome-results of a large prospective population-based study. Pain 2008; 137: 147.

14. Guillaume Found, Anderson Loundou, Nora Hamdani, et al. Anxiety and depression co-morbidities in irritable bowel syndrome (IBS): a systematic review and meta analysis.

15. Mariette Bengtosson, Klas Sjoberg, Martina Candamio et al. Anxiety in close relationships is higher and self esteem lower in patients with irritable bowel syndrome compared to patients with inflammatory bowel disease. European Journal of Internal Medicine. 2013; 24: 266-272.

16. Kabra N, Nadkarni A. Prevalence of depression and anxiety in irritable bowel syndrome: A clinic based study from India. Indian J Psychiatry. 2013 Jan; 55(1): 77-80.

17. Modabbernia MJ, Mansour-Ghanaei F, Imani A, MirsafaMoghaddam SA, Sedigh-Rahimabadi M, YousefiMashhour M, Joukar F, Atrkar-Roushan Z, Bidel S. Anxietydepressive disorders among irritable bowel syndrome patients in Guilan, Iran. BMC Res Notes. 2012 Feb 21; 5: 112.

18. Lee S, Wu J, Ma YL, Tsang A, Guo WJ, Sung J. Irritable bowel syndrome is strongly associated with generalized anxiety disorder: a community study. Aliment Pharmacol Ther. 2009 Sep 15; 30(6): 643-51.d 